\title{
Characterization of Monilinia fructicola Strains Resistant to Both Propiconazole and Boscalid
}

F. Chen and X. Liu, College of Agriculture and Biotechnology, China Agricultural University, Beijing 100193, China; S. Chen, College of Plant Science and Technology, Huazhong Agricultural University, Wuhan 430070, China; E. Schnabel, Department of Genetics and Biochemistry, Clemson University, Clemson, SC 29634; and G. Schnabel, School of Agricultural, Forest \& Environmental Sciences, Clemson University

\begin{abstract}
Chen, F., Liu, X., Chen, S., Schnabel, E., and Schnabel, G. 2013. Characterization of Monilinia fructicola strains resistant to both propiconazole and boscalid. Plant Dis. 97:645-651.

In 2011 and 2012, significant brown rot disease caused by Monilinia fructicola was observed in a peach orchard in Spartanburg County, SC, despite preharvest fungicide applications of demethylation inhibitor (DMI), quinone outside inhibitor (QoI), and succinate dehydrogenase inhibitor (SDHI) fungicides. All 22 isolates obtained in 2011 from this orchard were sensitive to the QoI fungicide, azoxystrobin, and the methyl benzimidazole carbamate (MBC) fungicide, thiophanatemethyl. Five were resistant to the DMI fungicide, propiconazole, and were selected, together with five propiconazole-sensitive isolates, for further investigations. One of the 10 isolates was resistant to propiconazole but sensitive to the SDHI fungicide, boscalid $\left(\mathrm{EC}_{50}=0.42\right.$ $\mu \mathrm{g} / \mathrm{ml}), 3$ were resistant to propiconazole with intermediate sensitivity to boscalid ( $\mathrm{EC}_{50} 0.72$ to $2.1 \mu \mathrm{g} / \mathrm{ml}$ ); 2 were sensitive to propiconazole with intermediate sensitivity to boscalid; 3 were sensitive to propiconazole but resistant to boscalid $\left(\mathrm{EC}_{50} \geq 2.1 \mu \mathrm{g} / \mathrm{ml}\right.$ ); and 1 (isolate MD22) was resistant to both propiconazole and boscalid. Disease incidence on detached fruit treated with formulated propiconazole or boscalid was significantly higher for MD22 compared to a sensitive control isolate.

Continued monitoring of fungicide resistance in the same orchard in 2012 revealed an increase of isolates resistant to propiconazole from $22.7 \%$ in 2011 to $34.7 \%$, and an increase of isolates resistant to both propiconazole and boscalid from $4.5 \%$ in 2011 to $18.4 \%$. Propiconazole resistance was always associated with the presence of the 'Mona' mobile element located upstream of the sterol $14 \alpha$-demethylase (MfCYP51) gene. To investigate whether mutations in the subunits of the succinate dehydrogenase enzyme were involved in boscalid resistance, significant portions of the M. fructicola $S d h A, S d h B, S d h C$, and $S d h D$ genes were cloned and analyzed for 2 sensitive, 2 boscalidresistant, and 6 dual-resistant isolates. Although sequence variation was found among the isolates, no single change correlated with resistance. Interestingly, analysis of isolates collected from orchards in 2001 and 2002, prior to the registration of boscalid, revealed a range of sensitivities to boscalid $\left(\mathrm{EC}_{50} 0.03\right.$ to $\left.3.46 \mu \mathrm{g} / \mathrm{ml}\right)$ including boscalid-resistant isolates. The presence of boscalid-resistant isolates in the baseline population was unexpected and requires further investigation.
\end{abstract}

Monilinia fructicola (Wint.) Honey causes brown rot blossom blight, twig canker, and fruit rot of stone fruits in the southeastern United States. Although sanitation practices such as pruning, removal of fruit mummies, and elimination of wild plum are widely implemented, these diseases still require fungicide applications for adequate control. Site-specific fungicides including benzimidazoles (MBCs; since the 1970s), sterol demethylation inhibitors (DMIs; since the 1980s), and more recently classes of respiration inhibiting fungicides including quinone outside inhibitors (QoIs) and succinate dehydrogenase inhibitors (SDHIs) have been used during bloom and/or preharvest to control blossom blight and fruit rot, respectively.

Site-specific fungicides generally are more effective compared to multisite fungicides, but they are prone to resistance development. Resistant field populations of fungi may emerge by means of selection following fungicide application. Resistance to DMIs in M. fructicola was first documented in Georgia and South Carolina (24), and later in Ohio, New York $(16,28)$, and New Jersey (5). Resistance is associated with a 65-bp inserted sequence designated 'Mona' located upstream of the $14 \alpha$-demethylase gene (MfCYP51). However, some isolates with reduced sensitivity to propiconazole from New York lacked Mona (28), indicating that other resistance

Corresponding authors: G. Schnabel, E-mail: schnabe@clemson.edu and X. Liu, E-mail: seedling@cau.edu.cn

Accepted for publication 6 December 2012.

http://dx.doi.org/10.1094/PDIS-10-12-0924-RE

(C) 2013 The American Phytopathological Society mechanisms may occur. Resistance to DMIs has yet to be documented on the West Coast. Reduced sensitivity to the SDHI boscalid and the QoI pyraclostrobin was observed in field isolates collected in 2008 from a single location in Spartanburg County, SC (1). The molecular basis of SDHI resistance in those isolates was not investigated because the nucleotide sequences of the succinate dehydrogenase subunit genes were unknown. The same study reported $\mathrm{EC}_{50}$ values for boscalid determined on potato dextrose medium; however, later studies identified minimal medium (MM) more suitable for determining sensitivity distributions to boscalid (11).

DMI and respiration inhibitor fungicides (which include QoIs and SDHIs) are rotated in the southeastern United States to control preharvest brown rot of peaches. Other chemical classes such as anilinopyrimidines and anilides are registered but not used during this critical time, mostly because of insufficient efficacy in the southeastern United States $(24,25)$. The emergence and spread of M. fructicola strains with dual resistance could have detrimental consequences for this disease management strategy. In 2011, heavy brown rot disease incidence was reported from a peach orchard in Spartanburg County, SC, the same orchard that revealed QoI and SDHI resistance 2 years earlier (1). The producer had used DMI and respiration inhibitor fungicides for blossom blight and preharvest brown rot control.

The objectives of this study were to determine the sensitivity of M. fructicola field isolates from Spartanburg County to azoxystrobin, boscalid, and propiconazole and to investigate the molecular mechanisms of resistance. In addition, the baseline sensitivity of $M$. fructicola isolates to boscalid was determined on MM for a group of isolates collected between 2001 and 2003 that, to our knowledge, had never been exposed to SDHI fungicides. 


\section{Materials and Methods}

Isolates. $M$. fructicola isolates were either retrieved from storage (isolates collected prior to 2011) or collected for this study from peach orchard MD located in Spartanburg County, SC, where brown rot disease was rampant between 2010 and 2012 despite preharvest applications of DMI and respiration inhibitor fungicides. Fifty-five isolates from storage collected in or prior to 2003 were used to determine the baseline sensitivity for boscalid on MM medium and had not been exposed to boscalid in the field to our knowledge. Those isolates were used in previous studies (24) and included 4, 10, 5, 5, 11, 6, and 14 isolates from Macon, Peach, Hall, and Crawford counties in Georgia and York, and Saluda and Spartanburg counties in South Carolina. All isolates came from commercial peach orchards, with the exception of DMI-resistant isolates Bmpc7 and 10, which were collected from the USDA Southeastern Fruit and Tree Nut Research Laboratory in Byron, GA. Isolates collected in 2011 (22 isolates) and 2012 (49 isolates) as well as isolate SC.dap3 (but not isolate SC.pdt9) were from orchard MD, about 2 ha in size and containing a mixture of at least 10 different peach and nectarine varieties, located in Spartanburg County.

The boscalid- and propiconazole-resistant phenotypes of isolates SCdap3, SC.pdt9, Bmpc7, and Bmpc10 (designated reference isolates) were determined in previous studies $(1,11,16,17)$. Isolates were recovered from stock cultures stored with silica blue gel beans at $-20^{\circ} \mathrm{C}$ on dried filter paper discs (Fisher Scientific, Pittsburgh, PA; Table 1). The isolates from storage were grown for 5 days on potato dextrose agar (PDA; Difco Laboratories, Sparks, $\mathrm{MD})$ before being used for experiments.
Fungicides. Commercial formulations of propiconazole (PropiMax EC, Dow Agro-Science LLC, Indianapolis, IN), boscalid (Endura, BASF, Research Triangle Park, NC), azoxystrobin (Abound, Syngenta Crop Protection, Greenville, NC), and thiophanate-methyl (Topsin-M, United Phosphorus, King of Prussia, PA) were used in this study. Products were dissolved in sterile water, adjusted to $5,000 \mu \mathrm{g} / \mathrm{ml}$, and stored at $4^{\circ} \mathrm{C}$. Salicylhydroxamic acid (SHAM) was added to azoxystrobin-amended PDA at $100 \mu \mathrm{g} / \mathrm{ml}$ to suppress the alternative oxidase pathway (22). Corresponding control dishes contained SHAM without azoxystrobin.

Determination of fungicide sensitivity. The distinction between isolates sensitive and resistant to propiconazole, azoxystrobin, and thiophanate-methyl was assessed on fungicideamended PDA (Difco Laboratories) at the discriminatory doses $0.3,1.0$, and $1.0 \mu \mathrm{g} / \mathrm{ml}$, respectively (23). Isolates were considered propiconazole-resistant when growth was greater than 20\%; azoxystrobin-resistant when growth was inhibited less than $50 \%$ (1); and thiophanate-methyl-resistant when growth was inhibited less than $50 \%(18)$. In previous studies $(11,30)$, propiconazoleresistant isolates were defined as growing more than $50 \%$ on 0.3 $\mu \mathrm{g} / \mathrm{ml}$ fungicide; however, sensitivity increases with each transfer and storage $(7,31)$, prompting us to decrease the threshold as described above. The sensitivity of isolates to boscalid in the form of $\mathrm{EC}_{50}$ values was determined as described previously (11). Boscalid was added to minimal medium (MM) at final concentrations of 0 , $0.05,0.1,0.5,1.0,5.0$, and $10.0 \mu \mathrm{g} / \mathrm{ml}$. Based on the ability to develop disease on boscalid-sprayed, detached peach fruit, isolates were considered boscalid-sensitive if $\mathrm{EC}_{50}$ values were $\leq 0.72$ $\mu \mathrm{g} / \mathrm{ml}$ (little disease incidence) and boscalid-resistant if the $\mathrm{EC}_{50}$ value was equal to or greater than $2.1 \mu \mathrm{g} / \mathrm{ml}$ (high disease inci-

Table 1. Origin and sensitivity of Monilinia fructicola isolates to propiconazole and boscalid

\begin{tabular}{|c|c|c|c|c|c|c|c|}
\hline \multirow[b]{2}{*}{ Isolate $^{x}$} & \multirow[b]{2}{*}{$\begin{array}{l}\text { No. of } \\
\text { isolates }\end{array}$} & \multirow[b]{2}{*}{ Origin } & \multicolumn{2}{|c|}{ Propiconazole $^{v}$} & \multirow[b]{2}{*}{$\begin{array}{c}\text { Presence/absence } \\
\text { of Mona }\end{array}$} & \multicolumn{2}{|c|}{ Boscalid $^{w}$} \\
\hline & & & $\begin{array}{c}\text { Mycelium growth } \\
(\%) \text { at } 0.3 \mu \mathrm{g} / \mathrm{ml}\end{array}$ & Phenotype & & $\begin{array}{c}\mathrm{EC}_{50} \\
(\mu \mathrm{g} / \mathrm{ml})\end{array}$ & Phenotype \\
\hline \multicolumn{8}{|l|}{ Reference isolates } \\
\hline SC.pdt9 & 1 & Orchard MD, SC & 0 & $\mathrm{~S}$ & - & 0.09 & $\mathrm{~S}$ \\
\hline Bmpc 10 & 1 & Byron, GA & 23 & $\mathrm{R}$ & + & 0.22 & $\mathrm{~S}$ \\
\hline Bmpc7 & 1 & Byron, GA & 25 & $\mathrm{R}$ & + & 0.30 & $\mathrm{~S}$ \\
\hline SC.dap3 & 1 & Orchard MD, SC & 23 & $\mathrm{R}$ & + & 2.89 & $\mathrm{R}$ \\
\hline \multicolumn{8}{|c|}{ Isolates collected in $2011(n=22)$} \\
\hline MD1 to 12 & 12 & Orchard MD, SC & 0 & $\mathrm{~S}$ & n.d. ${ }^{y}$ & n.d. & n.d. \\
\hline MD13 & 1 & Orchard MD, SC & 29 & $\mathrm{R}$ & + & 0.42 & $\mathrm{~S}$ \\
\hline MD14 & 1 & Orchard MD, SC & 33 & $\mathrm{R}$ & + & 1.22 & $\mathrm{IM}$ \\
\hline MD15 & 1 & Orchard MD, SC & 0 & $\mathrm{~S}$ & - & 1.34 & IM \\
\hline MD16 & 1 & Orchard MD, SC & 36 & $\mathrm{R}$ & + & 1.76 & IM \\
\hline MD17 & 1 & Orchard MD, SC & 32 & $\mathrm{R}$ & + & 1.89 & IM \\
\hline MD18 & 1 & Orchard MD, SC & 0 & $\mathrm{~S}$ & - & 2.04 & IM \\
\hline MD19 & 1 & Orchard MD, SC & 0 & $\mathrm{~S}$ & - & 2.67 & $\mathrm{R}$ \\
\hline MD20 & 1 & Orchard MD, SC & 0 & $\mathrm{~S}$ & - & 3.17 & $\mathrm{R}$ \\
\hline MD21 & 1 & Orchard MD, SC & 0 & $\mathrm{~S}$ & - & 4.37 & $\mathrm{R}$ \\
\hline MD22 & 1 & Orchard MD, SC & 44 & $\mathrm{R}$ & + & 11.94 & $\mathrm{R}$ \\
\hline \multicolumn{8}{|c|}{ Isolates collected in $2012(n=49)$} \\
\hline MD100 to 131 & 32 & Orchard MD, SC & 0 & $\mathrm{~S}$ & n.d. & n.d. & n.d. \\
\hline MD132 to 135 & 4 & Orchard MD, SC & $>30$ & $\mathrm{R}$ & + & $<0.33$ & $\mathrm{~S}$ \\
\hline MD136 to 139 & 4 & Orchard MD, SC & $>30$ & $\mathrm{R}$ & + & 1.1 to 1.7 & $\mathrm{IM}$ \\
\hline MD140 & 1 & Orchard MD, SC & $>30$ & $\mathrm{R}$ & + & 2.50 & $\mathrm{R}$ \\
\hline MD141 & 1 & Orchard MD, SC & $>30$ & $\mathrm{R}$ & + & 2.76 & $\mathrm{R}$ \\
\hline MD142 & 1 & Orchard MD, SC & $>30$ & $\mathrm{R}$ & + & 3.38 & $\mathrm{R}$ \\
\hline MD143 & 1 & Orchard MD, SC & $>30$ & $\mathrm{R}$ & + & 6.05 & $\mathrm{R}$ \\
\hline MD144 & 1 & Orchard MD, SC & $>30$ & $\mathrm{R}$ & + & 10.03 & $\mathrm{R}$ \\
\hline MD145 & 1 & Orchard MD, SC & $>30$ & $\mathrm{R}$ & + & 11.29 & $\mathrm{R}$ \\
\hline MD146 & 1 & Orchard MD, SC & $>30$ & $\mathrm{R}$ & + & 12.78 & $\mathrm{R}$ \\
\hline MD147 & 1 & Orchard MD, SC & $>30$ & $\mathrm{R}$ & $t^{\mathrm{z}}$ & 28.96 & $\mathrm{R}$ \\
\hline MD148 & 1 & Orchard MD, SC & $>30$ & $\mathrm{R}$ & + & 54.73 & $\mathrm{R}$ \\
\hline
\end{tabular}

$\mathrm{v}$ An isolate was considered propiconazole-resistant if the relative mycelial growth was $\geq 20 \%$ at the discriminatory dose of $0.3 \mu \mathrm{g} / \mathrm{ml}(16)$.

${ }^{\mathrm{w}}$ An isolate was considered sensitive $(\mathrm{S})$ to boscalid if the $\mathrm{EC}_{50}$ value was $\leq 0.72 \mu \mathrm{g} / \mathrm{ml}$, resistant (R) to boscalid if the EC 50 value was $\geq 2.10 \mu \mathrm{g} / \mathrm{ml}$, and intermediate (IM) if the $\mathrm{EC}_{50}$ value was $>0.72$ and $<2.10 \mu \mathrm{g} / \mathrm{ml}(1,11)$.

${ }^{x}$ All isolates were sensitive to azoxystrobin (data not shown).

y Not determined.

${ }^{\mathrm{z}}$ Sequence analysis revealed an uncharacterized insertion sequence about 1,200 bp in size just upstream of the Mona insertion. 
dence) (1,11). Isolates with $\mathrm{EC}_{50}$ values between 0.72 and 2.1 $\mu \mathrm{g} / \mathrm{ml}$ were considered intermediate because the resistance phenotype has not been sufficiently characterized. Sensitivity to tolnaftate was determined as described previously (13).

Detached fruit assay. To assess field relevance of dual resistant isolates, mature but firm commercial peach fruit were sprayed with label rates of formulated propiconazole or boscalid, inoculated, and assessed for disease incidence. Fruits were purchased from a local supermarket, washed two times with tap water to remove potential pesticide residues, and placed into plastic boxes until the surface had dried. Fungicides PropiMax EC (15 ml/liter) and Endura (1.34 $\mathrm{g} /$ liter) were sprayed to runoff onto the fruit $20 \mathrm{~h}$ prior to inoculation using a hand mister. Untreated fruit were sprayed with distilled sterile water. Three $4 \times 2 \mathrm{~mm}$ (diameter $\times$ depth) plugs were removed from each fruit at three equidistant locations $(2 \mathrm{~cm}$ apart) using a cork borer (4 $\mathrm{mm}$ diameter). The plugs were replaced within $1 \mathrm{~h}$ by mycelium plugs of equal dimension taken from the margins of 5-day-old cultures. After inoculation, the boxes were sealed with plastic bags to keep the relative humidity around $100 \%$ and maintained at room temperature. Disease incidence and lesion diameters were measured after 3 days. The experiment was conducted twice in a randomized complete block design with three replicates per experiment and three fruit in each replicate. Fitness parameters on detached fruit were conducted as described above, except that no fungicides were applied. Lesion diameters were recorded 3 days after inoculation.

Mycelial growth rate and conidia production in vitro. Mycelial plugs (4 $\mathrm{mm}$ diameter) taken from the periphery of 5-day-old cultures grown on $\mathrm{MM}$ were transferred to the center of dishes with MM. The dishes were incubated at $22^{\circ} \mathrm{C}$ in the dark. The colony diameter was measured after 4 days of incubation, with three replicates per isolate. Mean colony diameter divided by 4 was calculated for determination of daily growth rates. The experiment was conducted twice.

To assess the ability to produce spores, dishes with tomato sauce agar (227 g tomato sauce and $20 \mathrm{~g}$ agar in $750 \mathrm{ml}$ water) were inoculated with a 4-mm mycelial plug taken from the periphery of 4-day-old cultures and incubated for 10 days at $22^{\circ} \mathrm{C}$ in the dark. Conidia were washed off the medium with $30 \mathrm{ml}$ of distilled sterile water containing $0.1 \%$ Tween 20 . The suspension was transferred to $50-\mathrm{ml}$ tubes with 10 glass beads and vortexed for $1 \mathrm{~min}$. The conidia concentration in the suspension was estimated using a hemacytometer. Three replicate dishes were used per isolate and three assessments for each plate. The experiment was conducted twice.

Stability of resistance to boscalid. Isolates were recovered from storage at $4^{\circ} \mathrm{C}$ for 1 year. Mycelial plugs taken from the periphery of actively growing colonies were transferred weekly for a total of 5 weeks to fresh fungicide-free PDA petri dishes. $\mathrm{EC}_{50}$ values for boscalid were determined before the first and after the last transfer as described above.

Detection of the genetic mobile element Mona. Genomic DNA of $M$. fructicola was extracted from mycelium as described previously (6) with slight modifications. Briefly, the mycelium with spores was scraped off the surface of 4-day-old cultures grown on PDA and placed into 2-ml centrifuge tubes containing $500 \mu \mathrm{l}$ extraction buffer (1 M KCl, $100 \mathrm{mM}$ Tris-HCl, $10 \mathrm{mM}$ EDTA) with three glass beads $(425-600 \mu \mathrm{m}$, Sigma-Aldrich, St. Louis, MO). The tubes were vortexed for $1 \mathrm{~min}$ and centrifuged at 5,000 rpm for $10 \mathrm{~min}$. The supernatants were collected gently, transferred to new tubes, and mixed with $300 \mu \mathrm{l}$ 2-propanol (Sigma) by inverting the tube several times. The mixtures were centrifuged at 13,000 $\times g$ for $10 \mathrm{~min}$, and the pellets were dried and resuspended in $50 \mu \mathrm{l}$ TE (10 mM Tris- $\mathrm{HCl}$ and $1 \mathrm{mM}$ EDTA, $\mathrm{pH}$ 8.0) buffer.

Primer set INS65F (5'-TCGATGTTGCGTGGTCTGTCA-3') and INS65R (5'-CGGAGAGCGTCGATGTAGGTG5-3') (16) was used to detect the presence of Mona upstream of the MfCYP51 gene following published protocols (16). The PCR product was separated in a $1.0 \%$ agarose (Thermo Scientific, Rockford, IL) gel in $1 \times$ TAE buffer (40 mM Tris acetate and $1 \mathrm{mM}$ EDTA, $\mathrm{pH}$ 8.0).
Gels were photographed under UV light with an Electrophoresis Documentation and Analysis System (KODAK EDAS 290, Eastman Kodak Company, New Haven, CT).

Isolation of succinate dehydrogenase subunits from $M$. fructicola. Primers to amplify succinate dehydrogenase gene sequences from M. fructicola isolate DL15 were designed based on $S d h$ subunit gene sequences from the closely related species Botrytis cinerea and Sclerotinia sclerotiorum. This technique allowed amplification of an internal fragment of each subunit representing the majority of the gene. $S d h A, B, C$, and $D$ sequences were amplified using primer sets MfsdhaF (5'-AGAGCAGCATTCGGT CTTG-3') / MfsdhaR (5'-ACAGCCTTGCACTCATTCTC-3'), MfsdhbF (5'-CGCGACCAGCTTTCAGAAC-3') / MfsdhbR (5'ATTTCCTTCTTGATCTCCGC-3'), MfsdhcF (5'-GCAACTCAA CAATCGCTGC-3') / MfsdhcR (5'-AAAGCAACCAACGCCAAA G-3'), and MfsdhdF (5'-GTCGAAGCGTAGTTTCTCTAC-3') / MfsdhdR (5'-CTCATACAATCCAACTCCAACC-3'), respectively. These primers were used to amplify and sequence relevant subunits from 2 sensitive, 2 boscalid-resistant, and 6 dual-resistant isolates. The 50- $\mu$ l PCR reactions consisted of $1 \times$ PCR buffer, $50 \mathrm{ng}$ of DNA, $0.4 \mu \mathrm{M}$ of each primer, $100 \mu \mathrm{M}$ of each dNTP, and $3 \mathrm{U}$ of Taq DNA polymerase (New England BioLabs, Inc., Ipswich, MA). Amplifications were performed in an iCycler thermal cycler (BioRad Laboratories Co., Hercules, CA). Gene fragments were amplified with the following protocol: $94^{\circ} \mathrm{C}$ for $5 \mathrm{~min}$; followed by 32 cycles of $1 \mathrm{~min}$ at $94^{\circ} \mathrm{C} ; 40 \mathrm{~s}$ at $55^{\circ} \mathrm{C}(S d h A$ and $S d h C), 57^{\circ} \mathrm{C}$ $(S d h B)$, or $53^{\circ} \mathrm{C}(S d h D) ; 2.2 \mathrm{~min}(S d h \mathrm{~A}), 1 \mathrm{~min}(S d h \mathrm{~B})$, or $40 \mathrm{~s}$ $(S d h C$ and $S d h D)$ at $72^{\circ} \mathrm{C}$; and a final extension of $10 \mathrm{~min}$ at $72^{\circ} \mathrm{C}$. The PCR products were separated in $1.0 \%$ agarose gels in $1 \times \mathrm{TAE}$ buffer. PCR products were purified using the MinElute Gel Extraction kit (Qiagen Inc., Valencia, CA) or the ExoSAP-IT PCR purification kit (USB Corporation, Cleveland, $\mathrm{OH}$ ) following the instructions supplied by the manufacturer. Products were ligated into vector PCR4-Topo (Invitrogen Life Technologies, Grand Island, NY) prior to sequencing at the Clemson University Genomics Institute (CUGI). The sequencing primers were vector primers M13F and M13R, and MfsdhaF773 (5'-TGGTACTCTCCA CAGATTCC-3') located at position 773 in SdhA. DNA sequences were analyzed with DNAMAN7.0 software (Lynnon BioSoft, Quebec, Canada).

Data analysis. The $\mathrm{EC}_{50}$ value for each isolate was calculated by regression analysis using the percentage of growth inhibition against the logarithmic value of fungicide concentration. A statistical model was developed that related disease incidence and lesion size from detached fruit assays to the experimental replication, isolate, fungicide treatment, and their combination. The method of least squares was used to estimate the model terms associated with the factors, and analysis of variance was used to test for a significant effect of the factors on the response means. Mean separation was conducted using student $t$ test at $P=0.05$. Statistical analyses were performed using the statistical package JMP version 9.0.0 (2010 SAS Institute Inc., Cary, NC).

\section{Results}

Sensitivity of $M$. fructicola isolates to propiconazole, boscalid, azoxystrobin, and thiophanate-methyl. About $23 \%$ of the isolates (5 out of 22) collected in 2011 from orchard MD in Spartanburg County, SC were resistant to propiconazole as determined by reduced mycelial growth inhibition on PDA at the discriminatory dose of $0.3 \mu \mathrm{g} / \mathrm{ml}$ fungicide (Table 1 ). The $\mathrm{EC}_{50}$ values for boscalid were assessed on minimal medium for the 5 propiconazole-resistant and 5 randomly selected propiconazole-sensitive isolates and ranged from 0.42 to $11.94 \mu \mathrm{g} / \mathrm{ml}$. Four of those isolates (MD19 to MD22) had $\mathrm{EC}_{50}$ values higher than 2.1 and were therefore characterized as resistant $(1,11)$. Only reference isolate SC.dap3 and isolate MD22 were resistant to both propiconazole and boscalid (Table 1). The proportion of strains resistant to propiconazole only and to propiconazole and boscalid in orchard MD was determined in 2012. Thirty-five percent (17 isolates) were resistant to propiconazole, which represented a $12 \%$ increase over 
the previous year. Nine isolates (18.4\%) were resistant to both propiconazole and boscalid. All M. fructicola isolates collected in 2011 and 2012 were sensitive to azoxystrobin and thiophanatemethyl with the exception of two isolates from 2012 that were sensitive to boscalid and propiconazole but resistant to thiophanate-methyl (data not shown).

Assessment of fungicide performance on detached fruit inoculated with a sensitive or a dual resistant isolate. Isolate DL15, which was sensitive to propiconazole and boscalid in vitro, was completely controlled in both experiments on fruit treated with Propimax. Disease incidence on Endura-treated fruit was 17 and $11 \%$ with control efficacies of about $90 \%$ in the two experiments. In contrast, the dual resistant isolate MD22 caused $100 \%$ disease incidence in both experiments on fruit treated with Propimax or Endura with control efficacies of 4 and $35 \%$ for Propimax and 46 and 55\% for Endura (Table 2).

Fitness of isolates resistant to boscalid and propiconazole and stability of dual resistance. Three fitness components were investigated for 2 sensitive and 2 dual resistant isolates. No significant differences in ability to sporulate, growth of mycelium on MM, and lesion size on detached fruit was found between the two groups ( $P>0.05$ for all three fitness components; data not shown). Dual resistance was stable in both isolates after 1 year of storage on filter paper at $-20^{\circ} \mathrm{C}$ and after 5 weekly transfers on unamended PDA medium (data not shown).

Detection of the genetic mobile element Mona in dual-resistant isolates. Primer set INS65F and INS65R was used to screen for the presence of the Mona element upstream of the MfCYP51 gene (16). The primers amplified a 376-bp fragment in isolates carrying Mona and a 311-bp fragment in isolates without Mona. The 376-bp amplicon was obtained for all DMI-resistant isolates, except for isolate MD147. After increasing the extension time of the PCR reaction to 3 min per cycle, a fragment approximately $1,500 \mathrm{bp}$ in size was amplified from MD147. Sequence analysis revealed the existence of the Mona element and an additional, uncharacterized insertion sequence about 1,200 bp in size located $127 \mathrm{bp}$ upstream of the Mona element. The 311-bp amplicon was obtained for all DMI sensitive isolates (Table 1; Fig. 1).

Isolation of succinate dehydrogenase gene sequences from M. fructicola. DNA fragments of 1,814, 884, 688, and 577 bp in length were obtained from the $S d h A, S d h B, S d h C$, and $S d h D$ gene subunits of the boscalid-sensitive isolate DL15 using primer sets MfsdhaF/MfsdhaR, MfsdhbF/MfsdhbR, MfsdhcF/MfsdhcR, and MfsdhdF/MfsdhdR, respectively. The partial sequences covered a large percentage of the total gene sequences (Fig. 2) and contained all nucleotide positions known to correlate with boscalid resistance in other fungi (2). The fragments of subunits A to D contained 2, 1, 2 , and 2 introns, respectively, which varied in size between 51 and $87 \mathrm{bp}$. The coding sequences from the two isolates varied from each other at several positions including two sites producing amino acid differences in SdhA and SdhB. The deduced partial aa sequence of $S d h A$ was $98 \%$ identical to those of B. cinerea (GenBank accession number xp_001553708.1) and Sclerotinia sclerotiorum (xp_001591238.1), and 89 to $90 \%$ identical to those of Corynespora cassiicola (BAJ07109.1), Mycosphaerella gramini- cola (EGP92150.1), Paracoccidioides brasiliensis (EEH21556.1), Ajellomyces capsulatus (EEH04722.1), and Magnaporthe oryzae (xp_369076.2). The deduced partial aa sequence of $S d h B$ was 95 to 96\% identical to those of B. cinerea (xp_001548350.1) and S. sclerotiorum (xp_001594577.1) and 81 to $83 \%$ identical to those of Pyrenophora teres f. teres (xp_003302561.1), Alternaria alternata (ABW39788.1), and C. cassiicola (BAJ07110.1). The deduced partial aa sequence $S d h C$ was 88 to $90 \%$ identical to those of $S$. sclerotiorum (xp_001597467.1) and B. cinerea (xp_001547075.1) and 64 to $65 \%$ identical to those of Coccidioides posadasii (xp_003069888.1), Ajellomyces dematitidis (xp_002628383.1), and Penicillium chrysogenum (xp_002565350.1). The deduced partial aa sequence of $S d h D$ was $95 \%$ identical to that of $S$. sclerotiorum (xp_001593251.1), 89\% identical to that of B. cinerea (xp_001559774.1), and 60 to 66\% identical to those of Penicillium mameffei (xp_002146012.1), P. brasiliensis (xp_002792686.1), and Phaeosphaeria nodorum (xp_001801859.1). All above-mentioned sequences were the top hits of BLAST.

To assess for mutations that might be responsible for boscalid resistance, $S d h$ subunit gene sequences from four boscalid-resistant strains were analyzed. As was the case for the two control strains, nucleotide differences between the isolates were found (Table 3). At the sites of aa variation identified between the two control isolates, the resistant isolates all matched SC.pdt9 within SdhA and DL15 within SdhB. Two of the resistant isolates (MD20 and MD22) possessed no other predicted Sdh subunit changes. Three aa changes were predicted for isolate MD21 within SdhA, B, and $\mathrm{C}$ and one in SdhC of isolate SC.dap3. None of these aa changes are at positions that have previously been correlated with fungicide resistance. An additional 4 dual resistant isolates (MD141, MD145, MD147, and MD148) collected in 2012 were analyzed, but no aa variations were found in subunits $\mathrm{B}, \mathrm{C}$, and D (data not shown).

Baseline sensitivity of $M$. fructicola isolates to boscalid. To assess the natural variation in the sensitivity of $M$. fructicola to boscalid, the $\mathrm{EC}_{50}$ values of a collection of isolates that had never been exposed to boscalid was assayed. Among 55 isolates, values ranged from 0.03 to $3.46 \mu \mathrm{g} / \mathrm{ml}$ (Fig. 3). Most isolates $(n=45)$ had an $\mathrm{EC}_{50}$ value of less than $0.72 \mu \mathrm{g} / \mathrm{ml}$ and were classified as sensitive to boscalid. Surprisingly, however, four isolates had an $\mathrm{EC}_{50}$ value greater than $2.1 \mu \mathrm{g} / \mathrm{ml}$ and were considered resistant. The remain-

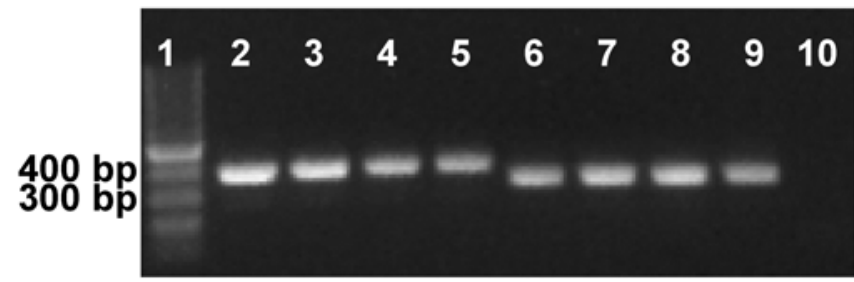

Fig. 1. Gel electrophoresis analysis of polymerase chain reaction (PCR) amplicons from Monilinia fructicola isolates amplified with primers INS65F and INS65R indicating the presence or absence of the Mona element. Lanes 1 to 10, low range plus DNA ladder (Fisher Scientific), propiconazole-resistant isolates Bmpc7, Bmpc10, dual resistant isolates MD22, SC.dap3, propiconazole-sensitive isolates MD20, MD21, SC.pdt9, DL15, and no DNA control, respectively.

Table 2. Disease incidence and control efficacy on detached fruit treated with propiconazole or boscalid formulated product for a sensitive and a dualresistant Monilinia fructicola isolate ${ }^{\mathrm{x}}$

\begin{tabular}{|c|c|c|c|c|c|c|}
\hline \multirow[b]{2}{*}{ Experiment } & \multirow[b]{2}{*}{ Isolate } & \multirow[b]{2}{*}{ Phenotype prop./bosc. ${ }^{y}$} & \multicolumn{2}{|c|}{ Propiconazole } & \multicolumn{2}{|c|}{ Boscalid } \\
\hline & & & Disease incidence & Control efficacy & Disease incidence & Control efficacy \\
\hline \multirow[t]{2}{*}{1} & DL15 & $\mathrm{S} / \mathrm{S}$ & $0 \mathrm{~b}^{\mathrm{z}}$ & $100 \mathrm{a}$ & $17 \mathrm{~b}$ & $87 \mathrm{a}$ \\
\hline & MD22 & $\mathrm{R} / \mathrm{R}$ & $100 \mathrm{a}$ & $4 \mathrm{~b}$ & $100 \mathrm{a}$ & $46 \mathrm{~b}$ \\
\hline \multirow[t]{2}{*}{2} & DL15 & $\mathrm{S} / \mathrm{S}$ & $0 \mathrm{~b}$ & $100 \mathrm{a}$ & $11 \mathrm{~b}$ & $96 \mathrm{a}$ \\
\hline & MD22 & $\mathrm{R} / \mathrm{R}$ & $100 \mathrm{a}$ & $35 \mathrm{~b}$ & $100 \mathrm{a}$ & $55 \mathrm{~b}$ \\
\hline
\end{tabular}

${ }^{x}$ Disease incidence was expressed as the percentage of wounds infected compared with the total number of wounds inoculated. Control efficacy was expressed as the percentage of the mean diameter of the control minus the mean diameter of the treatment divided by the mean diameter of the control. Propiconazole and boscalid were applied as PropiMax EC and Endura formulated products.

y $\mathrm{S}=$ sensitive and $\mathrm{R}=$ resistant to propiconazole/boscalid.

${ }^{\mathrm{z}}$ Values followed by the same letter within a column for each experiment separately are not significantly different according to student $t$ test at $P<0.05$. 
ing six isolates displayed an intermediate level of sensitivity to boscalid.

\section{Discussion}

This is the first reported case of resistance in M. fructicola, or any other Monilinia species, to both a DMI and a SDHI fungicide. The dual resistant isolates were collected from the same location but different years $(2008,2011$, and 2012); therefore the isolates could have derived from the same clonal population. It also indicates that this subpopulation is competitive and has survived and successfully competed for at least four consecutive years. Moreover, our results from 2012 indicate that the proportion of dual resistant isolates is increasing. In earlier studies, isolates from this location were sensitive to DMI fungicides but had reduced sensitivity to both boscalid and azoxystrobin (1). However, all isolates resistant to propiconazole and boscalid were sensitive to azoxystrobin, indicating that those phenotypes did not derive from a subpopulation resistant to both respiration inhibitors. In contrast, the presence of Mona in the dual-resistant isolates indicates that this genotype may have derived from a DMI-resistant population that was present but undetected in 2008 (16).

Various DMI fungicides and boscalid+pyraclostrobin in the form of Pristine formulated product (BASF Corp., Raleigh, NC) have been recommended to be used in rotation for the last decade to prevent preharvest brown rot of peach. This control strategy is not sustainable at this location any longer and may not be sustainable elsewhere if the dual-resistant subpopulation spreads to other orchards or emerges independently in other orchards. None of the dual-resistant isolates examined in this study or the study published previously was resistant to thiophanate-methyl, indicating that despite widespread resistance problems (23), MBCs may still be a viable component for spray programs trying to control populations with the above-described dual resistance. In addition, anilinopyrimidine and anilide fungicides may need to be reconsidered for preharvest brown rot management despite concerns about efficacy $(24,25)$.

An investigation into the resistance mechanism of dual-resistant isolates revealed that resistance to DMI fungicides was linked to the previously described, mobile genetic element Mona. The presence of Mona upstream of MfCYP51 causes over-expression of this gene (17). The presence of Mona correlated well with DMI fungicide resistance in isolates from South Carolina, Georgia, Ohio, New Jersey, and New York $(5,16)$, although some isolates with low to moderate DMI resistance lacked Mona (28).

We obtained sequences of the four previously uncharacterized Sdh subunits from $M$. fructicola isolates sensitive and resistant to boscalid and determined that the mechanism of resistance was not based on point mutations in any of the four Sdh subunits. In other organisms, resistance to boscalid has often been associated with a single mutation in Sdh subunits B, C, or D (3), whose residues in close proximity to the $[3 \mathrm{Fe}-4 \mathrm{~S}]$ cluster and heme $\mathrm{b}$ forms the ubiquinone binding sites $(10,26)$. The most common mutation for boscalid resistance occurs in Sdh subunit B, due to a $\mathrm{H} 277 \mathrm{Y}$ or $\mathrm{H} 277 \mathrm{R}$ aa change. Examples include resistance to boscalid in $A$. alternata (4), C. cassiicola (20), Podosphaera xanthii (21), and B. cinerea $(9,27,29)$. Some mutations linked to resistance were also

SdhA
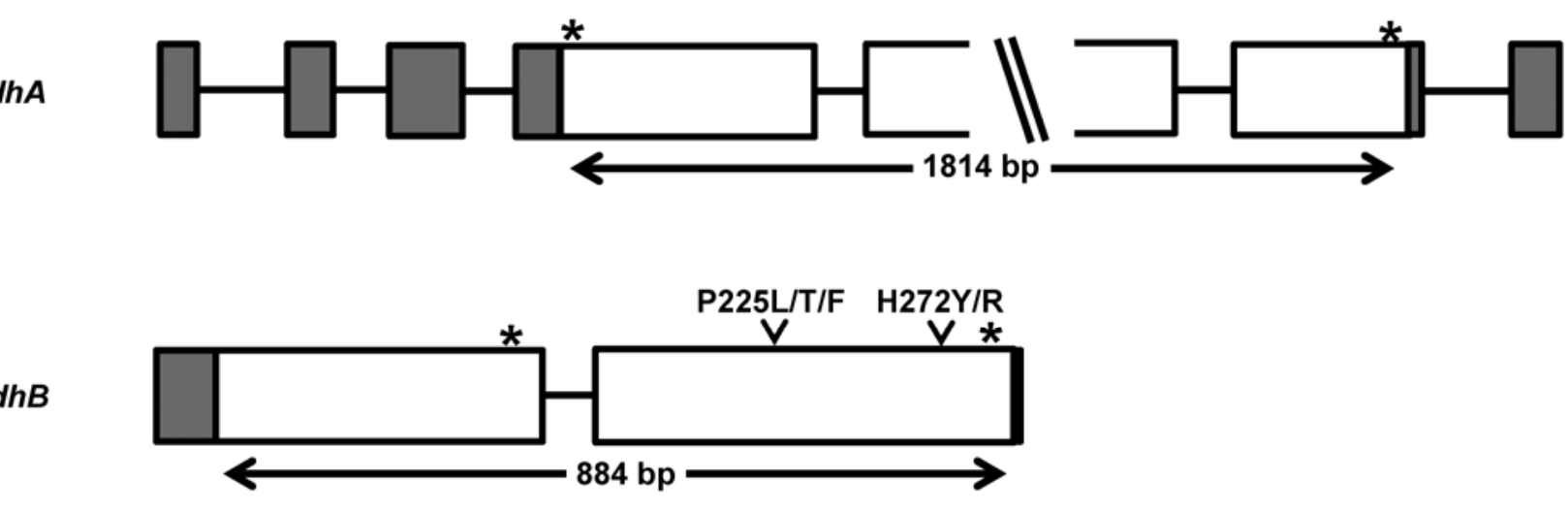

$\operatorname{SdhB}$
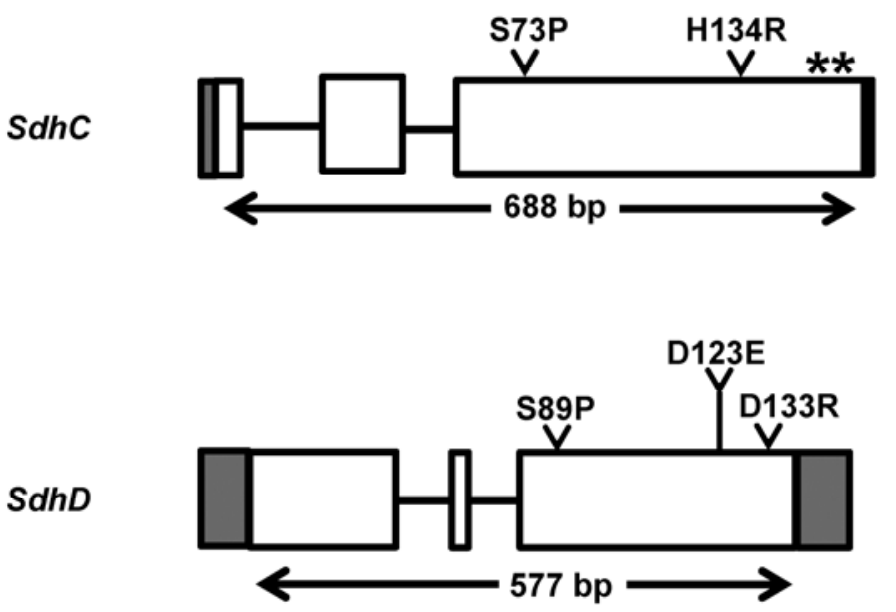

$100 \mathrm{bp}$

Fig. 2. Schematic of Sdh subunit genes in Monilinia fructicola indicating the regions cloned for this study. Solid lines indicate introns, white rectangles indicate cloned exons, and gray rectangles indicate noncloned exons. Noncloned intron and exon sequences were inferred from sequence homologies of Botrytis cinerea gene sequences retrieved from Broad Institute of MIT and Harvard (http://www.broadinstitute.org//scientific-community/data). Accession numbers for SdhA, SdhB, SdhC, and SdhD in B. cinerea are BC1G_07795.1, BC1G_13286.1, BC1G_14785.1, and BC1G_01333.1, respectively. The symbols "V" show the estimated location of the amino acid (aa) mutations identified to correspond with boscalid resistance in other studies, and the asterisks indicate aa variations not correlated with resistance identified in our study. The sequences for subunits A, B, C, and D were submitted to GenBank (accession numbers JQ436929, JQ436930, JQ436931, and JQ436932, respectively). The size of the gene fragments cloned from $M$. fructicola DNA is indicated below the gene structure. 
found in the Sdh subunits C and D $(2,15,20)$. Consistent with our study, C. cassiicola strains moderately resistant to boscalid did not reveal mutations in any of the four Sdh subunits (20). Those strains were harder to control in vivo, however, in studies using controlled conditions. As more SDHI fungicides are being registered, the sequences of Sdh subunits A to D cloned from M. fructicola will provide a valuable tool for future studies on the molecular mechanisms of SDHI resistance.

Since the resistance phenotype we were observing cannot be attributed to target site mutations, other mechanisms of resistance must be considered. Overexpression of membrane drug efflux proteins, such as ATP-binding cassette (ABC) and major facilitator superfamily (MFS) transporters, have been associated with resistance in B. cinerea (13), a close relative of $M$. fructicola. Both transporter types can mediate multidrug resistance (MDR) due to their low substrate specificity. MDR isolates of $B$. cinerea were categorized into three phenotypes (MDR1, -2, and -3) depending on specific tolerances to different classes of fungicides (13), and increased resistance to boscalid has been documented in MDR2 and MDR3 strains (15). One striking commonality among MDR isolates is that, in contrast to sensitive isolates, they all are resistant to tolnaftate, an inhibitor of squalene epoxidase. The $\mathrm{EC}_{50}$ values for tolnaftate of our dual-resistant isolates were 21 times higher than in sensitive $M$. fructicola isolates, indicating that MDR might indeed be involved (data not shown). This hypothesis is also supported by the fact that resistance levels of our dual resistant isolates to boscalid were not as high as the ones reported for isolates with single amino acid alterations in Sdh subunit genes (2). Generally, the resistance levels conferred by ABC and MFS transporters to solo fungicides are lower than those with target site mutations $(8,13)$.

In this study, we established the baseline sensitivity of $M$. fructicola isolates collected from nine locations in South Carolina and Georgia to boscalid, using relative mycelial growth on MM. Previously, the baseline sensitivity of $M$. fructicola isolates to boscalid was established on PDA (1), but we determined later that the separation of sensitive and resistant isolates is more pronounced on MM and established that MM is more suitable for testing sensitivity to boscalid in $M$. fructicola (11). The mean and median $\mathrm{EC}_{50}$ values of the baseline population of this study were 0.56 and 0.31 $\mu \mathrm{g} / \mathrm{ml}$, respectively. The mean $\mathrm{EC}_{50}$ reported for mycelial growth on PDA in M. fructicola was $2.23 \mu \mathrm{g} / \mathrm{ml}$ (1). The discrepancy can be explained by the different media used for sensitivity determination (11). The baseline $\mathrm{EC}_{50}$ values determined on $\mathrm{MM}$ will be useful for monitoring future shifts in sensitivity to boscalid in $M$. fructicola populations.

A small proportion $(7 \%)$ of the baseline isolates revealed $\mathrm{EC}_{50}$ values greater than $2.1 \mu \mathrm{g} / \mathrm{ml}$, indicating that phenotypes with reduced sensitivity may have existed prior to the introduction of boscalid. A similar proportion of isolates with reduced sensitivity was observed in A. alternata baseline isolates from pistachio orchards (4). If strains with reduced sensitivity to boscalid are common in wild type populations, as indicated in the two studies, it may explain why boscalid resistance is often observed after only a few years of selection pressure. For example, high frequencies of boscalid-resistant strains were reported in several important plant pathogens exposed to field rates of boscalid, such as Didymella bryoniae (12), B. cinerea $(9,14)$, and C. cassiicola (19). Three of the four M. fructicola isolates from the baseline population that were found to be resistant to boscalid were collected in 2001, 2

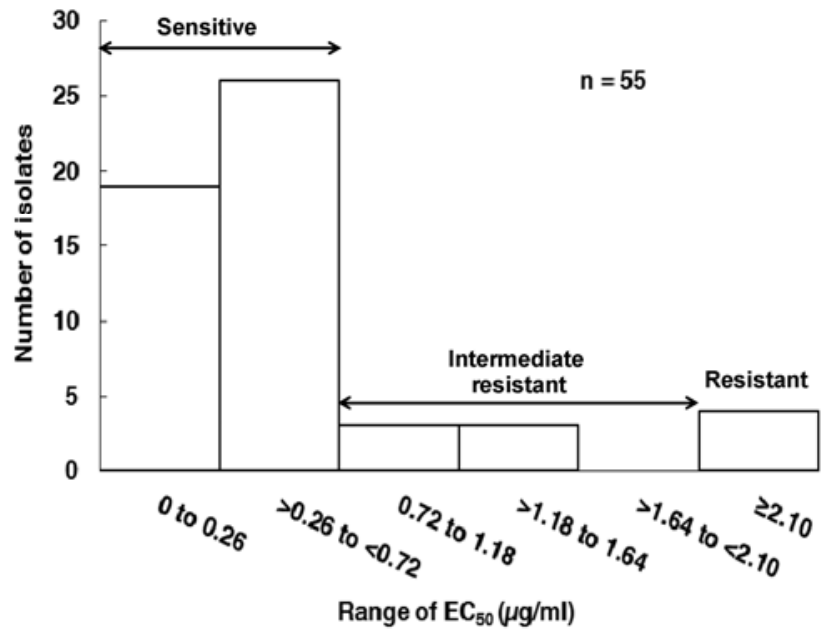

Fig. 3. Sensitivity of Monilinia fructicola baseline isolates to boscalid as determined by mycelium growth inhibition on minimal medium.

Table 3. Nucleotide mutations in succinate dehydrogenase (SDH) subunit exons from Monilinia fructicola

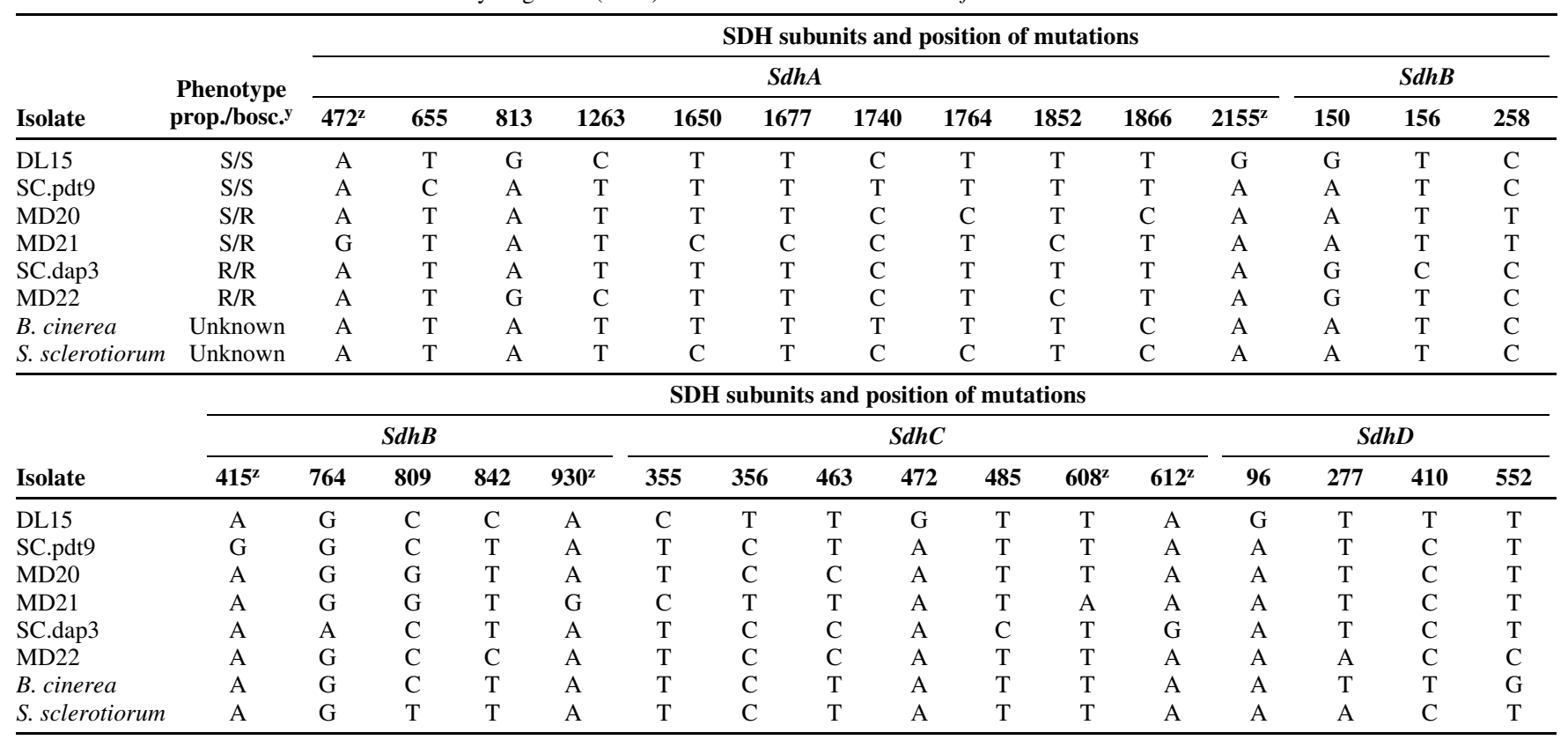

y $\mathrm{S}=$ sensitive and $\mathrm{R}=$ resistant to propiconazole/boscalid.

${ }^{\mathrm{z}}$ Mutations causing amino acid changes. The corresponding genetic codes ATA (472), CAA (2155), AAC (415), ATT (930), TGG (608), and GAT (612) changed to ATG, CGA, GAC, GTT, AGG, and GGT causing aa substitutions from I, Q, N, I, W, and D to M, R, D, V, R, and G, respectively. 
years prior to the first registration of Pristine, indicating that the isolates could not have originated from nearby, Pristine-treated orchards.

\section{Acknowledgments}

Technical contribution number 6044 of the Clemson University Experiment Station. This material is based upon work supported by the CSREES/USDA, under project number SC-1000642, the USDA Grants Contract no. 2010-3410321000 and 2009-41534-05445, and the USDA NIFA Extension IPM Program. We thank P. K. Bryson (Clemson University, Clemson, USA) for technical support.

\section{Literature Cited}

1. Amiri, A., Brannen, P. M., and Schnabel, G. 2010. Reduced sensitivity in Monilinia fructicola field isolates from South Carolina and Georgia to respiration inhibitor fungicides. Plant Dis. 94:737-743.

2. Avenot, H., Sellam, A., and Michailides, T. 2009. Characterization of mutations in the membrane-anchored subunits AaSDHC and AaSDHD of succinate dehydrogenase from Alternaria alternata isolates conferring field resistance to the fungicide boscalid. Plant Pathol. 58:1134-1143.

3. Avenot, H. F., and Michailides, T. J. 2010. Progress in understanding molecular mechanisms and evolution of resistance to succinate dehydrogenase inhibiting (SDHI) fungicides in phytopathogenic fungi. Crop Prot. 29:643651.

4. Avenot, H. F., Sellam, A., Karaoglanidis, G., and Michailides, T. J. 2008. Characterization of mutations in the iron-sulphur subunit of succinate dehydrogenase correlating with boscalid resistance in Alternaria alternata from California pistachio. Phytopathology 98:736-742.

5. Burnett, A., Lalancette, N., and McFarland, K. 2010. First report of the peach brown rot fungus Monilinia fructicola resistant to demethylation inhibitor fungicides in New Jersey. Plant Dis. 94:126.

6. Chi, M., Park, S., and Lee, Y. 2009. A quick and safe method for fungal DNA extraction. J. Plant Pathol. 25:108-111.

7. Cox, K. D., Bryson, P. K., and Schnabel, G. 2007. Instability of propiconazole resistance and fitness in Monilinia fructicola. Phytopathology 97:448-453.

8. de Waard, M. A., Andrade, A. C., Hayashi, K., Schoonbeek, H. J., Stergiopoulos, I., and Zwiers, L. H. 2006. Impact of fungal drug transporters on fungicide sensitivity, multidrug resistance and virulence. Pest Manag. Sci. 62:195-207.

9. Fernández-Ortuño, D., Chen, F., and Schnabel, G. 2012. Resistance to pyraclostrobin and boscalid in Botrytis cinerea isolates from strawberry fields in the Carolinas. Plant Dis. 96:1198-1203.

10. Horsefield, R., Yankovskaya, V., Sexton, G., Whittingham, W., Shiomi, K., Omura, S., Byrne, B., Cecchini, G., and Iwata, S. 2006. Structural and computational analysis of the quinone-binding site of complex II (succinateubiquinone oxidoreductase) - A mechanism of electron transfer and proton conduction during ubiquinone reduction. J. Biol. Chem. 281:7309-7316.

11. Hu, M., Luo, C., Grabke, A., and Schnabel, G. 2011. Selection of a suitable medium to determine sensitivity of Monilinia fructicola mycelium to SDHI fungicides. J. Phytopathol. 159:616-620.

12. Keinath, A. P. 2012. Differential sensitivity to boscalid in conidia and ascospores of Didymella bryoniae and frequency of boscalid-insensitive isolates in South Carolina. Plant Dis. 96:228-234.

13. Kretschmer, M., Leroch, M., Mosbach, A., Walker, A., Fillinger, S., Mernke, D., Schoonbeek, H., Pradier, J., Leroux, P., De Waard, M. A., and Hahn, M. 2009. Fungicide-driven evolution and molecular basis of multidrug resistance in field populations of the grey mould fungus Botrytis cinerea. Plos Pathog. 5:e1000696.
14. Leroch, M. K. M. a. H. M. 2011. Fungicide resistance phenotypes of Botrytis cinerea isolates from commercial vineyards in South West Germany. J. Phytopathol. 159:63-65.

15. Leroux, P., Gredt, M., Leroch, M., and Walker, A. S. 2010. Exploring mechanisms of resistance to respiratory inhibitors in field strains of Botrytis cinerea, the causal agent of gray mold. Appl. Environ. Microbiol. 76:66156630.

16. Luo, C., Cox, K. D., Amiri, A., and Schnabel, G. 2008. Occurrence and detection of the DMI resistance-associated genetic element 'Mona' in $\mathrm{Mo}$ nilinia fructicola. Plant Dis. 92:1099-1103.

17. Luo, C., and Schnabel, G. 2008. The cytochrome p450 lanosterol 14 alphademethylase gene is a demethylation inhibitor fungicide resistance determinant in Monilinia fructicola field isolates from Georgia. Appl. Environ. Microbiol. 74:359-366.

18. Ma, Z. H., Luo, Y., and Michailides, T. 2006. Molecular characterization of the two-component histidine kinase gene from Monilinia fructicola. Pest Manag. Sci. 62:991-998.

19. Miyamoto, T., Ishii, H., Seko, T., Kobori, S., and Tomita, Y. 2009. Occurrence of Corynespora cassiicola isolates resistant to boscalid on cucumber in Ibaraki Prefecture, Japan. Plant Pathol. 58:1144-1151.

20. Miyamoto, T., Ishii, H., Stammler, G., Koch, A., Ogawara, T., Tomita, Y., Fountaine, J. M., Ushio, S., Seko, T., and Kobori, S. 2010. Distribution and molecular characterization of Corynespora cassiicola isolates resistant to boscalid. Plant Pathol. 59:873-881.

21. Miyamoto, T., Ishii, H., and Tomita, Y. 2010. Occurrence of boscalid resistance in cucumber powdery mildew in Japan and molecular characterization of the iron-sulfur protein of succinate dehydrogenase of the causal fungus. J. Gen. Plant Pathol. 76:261-267.

22. Olaya, G., Zheng, D. S., and Koller, W. 1998. Differential responses of germinating Venturia inaequalis conidia to kresoxim-methyl. Pest Manag. Sci. 54:230-236.

23. Schnabel, G., Amiri, A., and Brannen, P. 2011. Field kit- and internet-supported fungicide resistance monitoring. Pages 116-131 in: Fungicide Resistance in Crop Protection: Risk and Management. T. S. Thind, ed. CAB International, Cambridge, MA.

24. Schnabel, G., Bryson, P. K., Bridges, W. C., and Brannen, P. M. 2004. Reduced sensitivity in Monilinia fructicola to propiconazole in Georgia and implications for disease management. Plant Dis. 88:1000-1004.

25. Schnabel, G., Hudson, S. B., and Bridges, W. C. 2009. Preharvest fungicide programs for pre- and postharvest brown rot control in peach and nectarine. Plant Dis. Manag. Rep. 3:STF002.

26. Sun, F., Huo, X., Zhai, Y. J., Wang, A. J., Xu, J. X., Su, D., Bartlam, M., and Rao, Z. H. 2005. Crystal structure of mitochondrial respiratory membrane protein complex II. Cell 121:1043-1057.

27. Veloukas, T., Leroch, M., Hahn, M., and Karaoglanidis, G. S. 2011. Detection and molecular characterization of boscalid-resistant Botrytis cinerea isolates from strawberry. Plant Dis. 95:1302-1307.

28. Villani, S. M., and Cox, K. D. 2011. Characterizing fenbuconazole and propiconazole sensitivity and prevalence of 'Mona' in isolates of Monilinia fructicola from New York. Plant Dis. 95:828-834.

29. Yin, Y. N., Kim, Y. K., and Xiao, C. L. 2011. Molecular characterization of boscalid resistance in field isolates of Botrytis cinerea from apple. Phytopathology 101:986-995.

30. Zehr, E. I., Luszcz, L. A., Olien, W. C., Newall, W. C., and Toler, J. E. 1999. Reduced sensitivity in Monilinia fructicola to propiconazole following prolonged exposure in peach orchards. Plant Dis. 83:913-916.

31. Zhu, F., Bryson, P. K., and Schnabel, G. 2012. Influence of storage approaches on instability of propiconazole resistance in Monilinia fructicola. Pest Manag. Sci. 68:1003-1009. 